\title{
IMPACTO DA ALTERAÇÃO DA COBERTURA DO SOLO NOS PARÂMETROS BIOFÍSICOS NO SUL DA FLORESTA AMAZÔNICA POR SENSORIAMENTO REMOTO
}

\author{
ROTHMUND, Lucas Douglas - lucaspgfa@fisica.ufmt.br \\ Universidade Federal de Mato Grosso / UFMT \\ ALMEIDA JÚNIOR, Elio Santos - elio_jr_@hotmail.com \\ Universidade Federal de Mato Grosso / UFMT \\ LIMA, Luis Philippe de Arruda - luisphilippe@fisica.ufmt.br \\ Universidade Federal de Mato Grosso / UFMT \\ MASSAD, Heloisa Agnes Bodnar - heloisamassad@gmail.com \\ Universidade Federal de Mato Grosso / UFMT \\ PALÁCIOS, Rafael da Silva - rafael.pgfa@gmail.com \\ Universidade Federal de Mato Grosso / UFMT \\ BIUDES, Marcelo Sacardi - marcelo@fisica.ufmt.br \\ Universidade Federal de Mato Grosso / UFMT \\ MACHADO, Nadja Gomes - nadja.machado@blv.ifmt.edu.br \\ Instituto Federal de Mato Grosso / IFMT \\ NOGUEIRA, José de Souza - nogueira@ufmt.br \\ Universidade Federal de Mato Grosso / UFMT
}

\begin{abstract}
RESUMO: A Floresta Amazônica tem grande importância ambiental, contribuindo na manutenção da biodiversidade, no sequestro de carbono e na ciclagem de água, entretanto, as transformações decorrentes das atividades antrópicas alteram a forma como a radiação solar interage com a superfície terrestre. O objetivo do trabalho foi avaliar a influência nos parâmetros biofísicos albedo, Normalized Difference Vegetation Index (NDVI), temperatura de brilho e saldo de radiação em virtude da conversão de uma área de Floresta Amazônica em pastagem, para os períodos seco e chuvoso no noroeste de Mato Grosso utilizando a técnica de sensoriamento remoto. Foram utilizadas imagens do sensor OLI (Operatinal Land Imager) a bordo do satélite Landsat-8, adquiridas nos meses de abril e outubro de 2016, referentes aos períodos chuvoso e seco respectivamente. A substituição da área de Floresta Amazônica em pastagem influenciou os parâmetros biofísicos da superfície, devido à alteração na estrutura e nas propriedades ópticas da superfície. A floresta nativa apresentou os maiores valores de NDVI em relação a pastagem. $O$ albedo e a temperatura de brilho da superfície apresentaram valores inversos ao NDVI, com menores valores para a floresta nativa. A substituição da Floresta Amazônica em pastagem diminuiu a energia disponível à superfície, tendo o saldo de radiação apresentado os menores valores para a pastagem.
\end{abstract}

PALAVRAS-CHAVES: Desmatamento; Análise microclimática; Imagem de satélite; NDVI; Albedo.

IMPACT OF SOIL COVERAGE CHANGE ON BIOPHYSICAL PARAMETERS OF THE SOUTHERN AMAZON FOREST BY REMOTE SENSING

ABSTRACT: The Amazon rainforest has great environmental importance, contributing to the maintenance of biodiversity, carbon sink and water cycling, however, the changes arising from human activities modify the way the solar radiation interacts with the Earth's surface. The objective of this work was to evaluate the influence on biophysical 
parameters albedo, NDVI, brightness temperature and net radiation due to the conversion of an area of the Amazon rainforest in pasture, for dry and wet periods in northwestern Mato Grosso using remote sensing technique. Where used Sensor images from OLI (Operatinal Land Imager) aboard the Landsat satellite-8, acquired in the months of April and October 2016 for the rainy and dry periods respectively. The replacement of the Amazon forest area in pasture influenced the biophysical parameters of the surface, due to the change in structure and optical properties of the surface. The native forest presented the highest values of NDVI in relation to the pasture. The albedo and surface temperature presented inverse values to the NDVI, with lower values for the native forest. The replacement of the Amazon forest into pasture reduced the available energy to the surface, taking the balance of radiation presented the lowest values in the modified area.

KEYWORDS: Deforestation; Microclimatic analysis; Satellite image; NDVI; Albedo

\section{INTRODUÇÃO}

A Amazônia possui aproximadamente 6,7 milhões $\mathrm{km}^{2}$, e é considerada a maior floresta tropical do mundo, onde $60 \%$ desta área encontra-se em território brasileiro, distribuída entre os estados do Acre, Amapá, Amazonas, Pará, Rondônia e Roraima, Maranhão, Tocantins e Mato Grosso (GUERRA, 2011; ARPA, 2012). Além disso, apresenta grande importância econômica e ambiental para o país, com destaque principalmente na manutenção da biodiversidade, no sequestro de carbono, na ciclagem de água e manutenção da biodiversidade (ROCHA et al., 2015).

Apesar da enorme relevância existente, a Floresta Amazônica vem sofrendo com mudanças estruturais, especialmente durante o último século, sobretudo devido a atividades antrópicas, como a conversão de áreas florestadas em manejadas, terras agrícolas e pastagens (FAUSTO et al., 2016; LIMA, 2016). A substituição da cobertura natural modifica a forma de interação da radiação solar com a superfície, podendo impactar diretamente o microclima local e, a longo prazo, o clima regional e global (BERBET, 2002; BERNARDES et al., 2011; SILVA et al., 2011). Este impacto tem sido motivo de estudo em diversas pesquisas, o que é de grande importância, contribuindo na compreensão das mudanças e um melhor entendimento dos sistemas naturais (LIBERATO, 2011; SILVA et al., 2015; GUILHERME et al., 2016).

O mapeamento da cobertura vegetal pode ser realizado de diferentes formas, comumente são realizadas visitas in loco para aquisição de dados, entretanto, esta abordagem apresenta uma área de abrangência reduzida e sua espacialização é onerosa (PAVÃO et al., 2015). Uma alternativa de uso crescente é o emprego de imagens de satélite, o que oportuniza um monitoramento contínuo, com uma maior área de abrangência e a redução de custos na obtenção dos dados, facilitando assim o desenvolvimento de novos estudos (PEREIRA et al., 2007).

Em virtude de diferentes respostas espectrais que se obtém em relação aos distintos tipos de cobertura e ecossistemas, até mesmo em áreas heterogêneas, as técnicas e ferramentas de sensoriamento remoto permitem a estimativa de diferentes parâmetros biofísicos com elevado grau de acurácia (TEIXEIRA et al., 2009; LOPES et al., 2010; BEZERRA et al., 2014). Como, por exemplo, uma análise multi-temporal das mudanças na cobertura do solo empregando o Índice de Vegetação da Diferença Normalizada (NDVI), Índice de Área Foliar (LAI) e o Índice de Vegetação Melhorado (EVI), correlacionando as estimativas de albedo, temperatura da superfície e saldo de radiação, pode 
evidenciar a forte correlação entre o albedo e o tipo de cobertura do solo, além de validar o emprego de imagens de satélite para esta finalidade (CUNHA et al., 2012).

Avaliar os parâmetros biofísicos por meio de imagens de satélite, nos permite identificar alterações ocorridas na superfície, além de poder caracterizar sua influência nos índices biofísicos (OLIVEIRA et al., 2012; BEZERRA et al., 2014; MARTINS et al., 2015; RIBEIRO et al., 2015). Desta maneira, o objetivo do presente trabalho foi avaliar as alterações no NDVI, albedo, temperatura de brilho e saldo de radiação por técnicas de sensoriamento remoto devido à conversão de uma área de floresta nativa em pastagem no Sul da Amazônia, em Colniza, Mato Grosso.

\section{MATERIAL E MÉTODOS}

\subsection{DESCRIÇÃO DA ÁREA DE ESTUDO}

A área de estudo está localizada no município de Colniza, noroeste do estado de Mato Grosso, 1.035 km distante da capital Cuiabá (MT). O local de estudo possui aproximadamente 50 ha e coordenadas centrais de latitude $9^{\circ} 36^{\prime}$ S e longitude $59^{\circ} 24^{\prime} \mathrm{O}$, com altitude média de $150 \mathrm{~m}$ e $11 \mathrm{Km}$ de distância da cidade. A área de estudo é constituída de dois talhões distintos e em dimensões semelhantes, de floresta nativa e pastagem como pode ser observado na Figura 1.

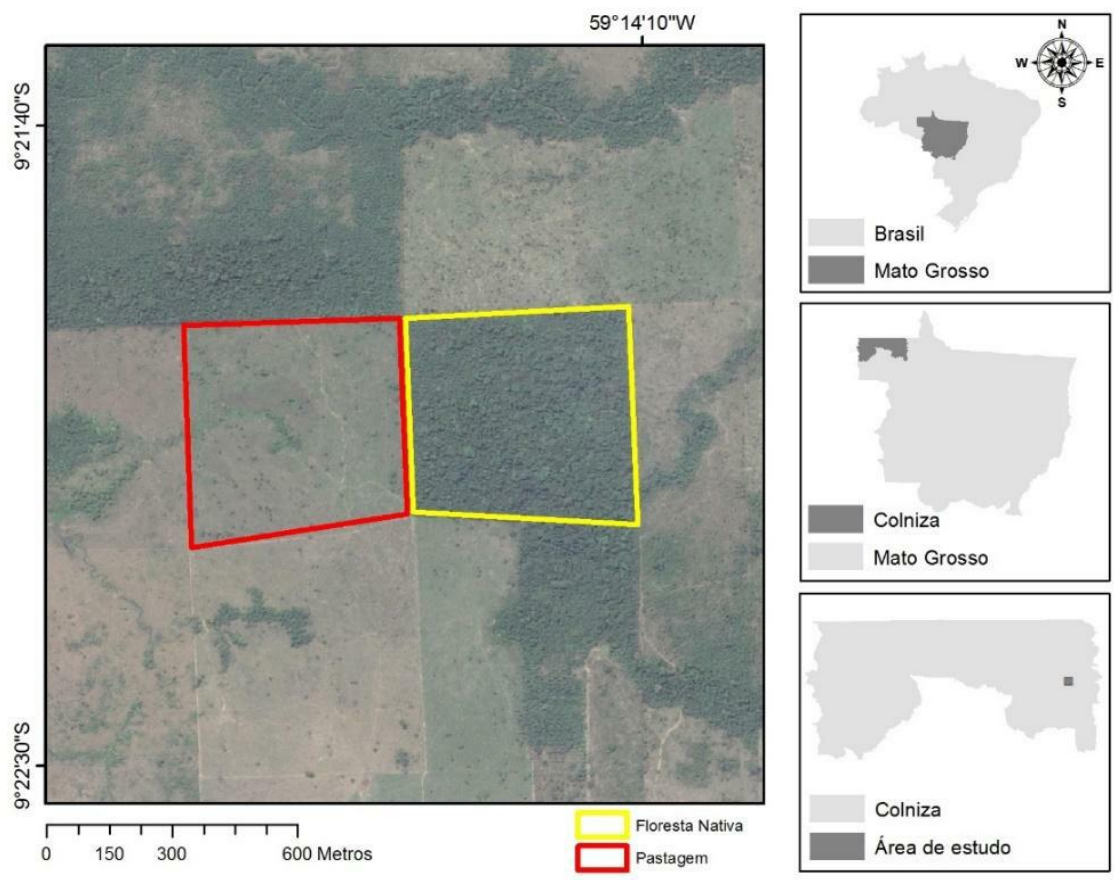

Figura 1- Localização da área de estudo no município de Colniza, Mato Grosso.

Segundo a classificação de Köppen o clima na região é Am, com temperatura média do mês mais frio superior a $18^{\circ} \mathrm{C}$ e precipitação anual média de 2800 a $3100 \mathrm{~mm}$. A região apresenta um período chuvoso entre os meses de 
novembro a abril, e seco de pequena duração entre os meses de junho a setembro, sendo os meses de maio e outubro considerados meses de transição (ALVARES et al., 2013; TRES et al., 2016).

\subsection{IMAGENS DE SATÉLITE}

Foram utilizadas imagens obtidas por meio dos sensores Operational Land Imager (OLI) e Thermal Infrared Sensor (TIRS), a bordo do satélite Landsat-8, ponto 67 e órbita 229, disponibilizadas por meio do United States Geological Survey - USGS para as datas de 28/04/2016, referente ao período chuvoso, e 17/10/2016 referente ao período seco.

\subsection{DADOS DE SUPERFÍCIE}

A cidade de Colniza, que não apresenta disponibilidade de dados meteorológicos ou micrometeorológicos oriundos de estação pertencentes ao INMET (Instituto Nacional de Meteorologia), situa-se na área de cobertura da estação meteorológica da cidade de Cotriguaçu-MT (90 Km de distância do local de estudo). Por isso, os dados de temperatura do ar $\left({ }^{\circ} \mathrm{C}\right)$ e de umidade relativa do ar (\%), aplicados ao modelo (seção 2.4), que foram obtidos por meio da plataforma eletrônica do INMET, referem-se à cidade de Cotriguaçu. Esse critério utilizado está de acordo com o manual da Organização Meteorológica Mundial (OMM) n.o 544, 2003, no qual estabelece que o intervalo horizontal entre estações, em geral, não deve exceder $300 \mathrm{~km}$ em regiões pouco povoadas (WMO, 2003).

\subsection{ESTIMATIVA DOS PARÂMETROS BIOFÍSICOS}

Para obtenção dos parâmetros biofísicos foi utilizado a parte inicial do modelo SEBAL, que consiste na obtenção do saldo de radiação (Equação 7). Além disso, utilizou-se do Índice de Vegetação da Diferença Normalizada (Normalized Difference Vegetation Index - NDVI), que foi obtido pela razão entre a diferença e a soma das refletâncias das bandas $\rho_{5}$ e $\rho_{4}$ do OLI Landsat-8 (Equação 1). Esse índice atua como indicador sensível à quantidade e às atividades fenológicas da vegetação, variando de -1 a 1 (PISANI et al. 2012; PONZONI \& SHIMABUKURO, 2009).

$$
N D V I=\frac{\left(\rho_{5}-\rho_{4}\right)}{\left(\rho_{5}+\rho_{4}\right)}
$$

O albedo da superfície $\alpha_{\text {sup }}$ foi obtido pela Equação (2).

$$
\alpha_{\text {sup }}=\frac{\alpha_{t o a}-\alpha_{a t m}}{\tau_{w}^{2}}
$$

Onde $\alpha_{t o a}$ é o albedo do topo da atmosfera (Equação 3), $\alpha_{a t m}$ é a radiação solar média refletida pela atmosfera antes de alcançar a superfície, considerada nesse trabalho igual a 0,03 (PELGRUM et al., 2005), e $\tau_{w}$ é a transitividade atmosférica (Equação 4). 


$$
\alpha_{\text {toa }}=0.300 \rho_{2}+0.277 \rho_{3}+0.233 \rho_{4}+0.143 \rho_{5}+0.036 \rho_{6}+0.012 \rho_{7}
$$

Onde $\rho_{2}, \rho_{3}, \rho_{4}, \rho_{5}, \rho_{6}$ e $\rho_{7}$ são as refletâncias espectrais das bandas $2,3,4,5,6$ e 7 do sensor OLI do Landsat-8.

$$
\tau_{w}=0,35+0,627 e^{\left[\frac{-0,00146 P}{K_{t} \cos \theta}-0,075\left(\frac{W}{\cos \theta}\right)^{0,4}\right]}
$$

Onde $W$ é a água precipitável (Equação 5 ), $P$ a pressão atmosférica (Equação $6), K_{t}$ a turbidez da atmosfera, $\theta$ é o ângulo zenital no momento da passagem do satélite.

$$
W=0,14 e_{a} P+2,1
$$

Onde $P$ é a pressão atmosférica (Equação 6 ) e $e_{a}$ é a pressão atual de vapor d'água.

$$
P=101,3\left(\frac{T_{a r}-0,0065 z}{T_{a r}}\right)^{5,26}
$$

Onde $T_{a r}$ é a temperatura do ar e $z$ é a altitude média da superfície em relação ao nível do mar.

O saldo de radiação instantâneo, no momento da passagem do satélite, foi calculado segundo a Equação (7).

$$
R n=\left(1-\alpha_{\text {sup }}\right) R c_{\text {sol,inc }}+R l_{o l, a t m}-R l_{o l, e m i t}-\left(1-\varepsilon_{a}\right) R l_{o l, a t m}
$$

Onde $R c_{\text {sol,inc }}$ é o fluxo de radiação solar que atinge a superfície terrestre (Equação 8), $R l_{o l, a t m}$ é a radiação de onda longa emitida pela atmosfera (Equação 9), $R l_{o l, e m i t}$ é a radiação de onda longa emitida pela superfície terrestre (Equação 10 ) e, por fim, $\alpha_{\text {sup }}$ é o albedo de superfície calculado pela Equação (2).

$$
R c_{\text {sol,inc }}=S . \operatorname{Cos} Z \cdot d_{r} \cdot \tau_{s w}
$$

Onde S é a constante solar $\left(1367 \mathrm{Wm}^{-2}\right), Z$ é o ângulo zenital solar, $d_{r}$ representa o inverso do quadrado da distância relativa Terra-Sol e $\tau_{s w}$ é a transmissividade atmosférica.

$$
R l_{o l, a t m}=\varepsilon_{a} \cdot \sigma \cdot T_{a}^{4}
$$

Onde $\varepsilon_{a}$ é a emissividade da atmosfera (Equação 11) (DUARTE et al., 2006), $\sigma$ é a constante de Stefan-Boltzman cujo valor é $5,67 \cdot 10^{-8} \mathrm{Wm}^{-2} \mathrm{~K}^{-4}$ e $T_{a}$ é a temperatura do ar. 


$$
R l_{o l, e m i t}=\varepsilon_{0} \cdot \sigma \cdot T^{4}
$$

Onde $\varepsilon_{0}$ representa a emissividade de cada pixel, $\sigma$ representa a constante de Stefan-Boltzman e $T_{a}$ é a temperatura da superfície.

$$
\varepsilon_{a}=0,625 \cdot\left(\frac{e_{a}}{T_{a}}\right)^{0,131}
$$

Onde $e_{a}$ representa a pressão de vapor e $T_{a}$ a temperatura do ar.

\subsection{AMOSTRAGEM E ANÁLISE ESTATÍSTICA}

A amostragem para análise dos dados consistiu na obtenção dos valores de 18 pixels, distribuídos em formato de grade, como pode ser visto na Figura 2. Os pixels escolhidos para análise foram nomeados de $A$ a $R$, sendo 9 para a região de Pastagem ( $A$ a $I$ ) e 9 para a região de Floresta Nativa ( $J$ a $R$ ). $O$ processo de amostragem descrito foi utilizado para cada parâmetro biofísico e repetido em ambas as estações.

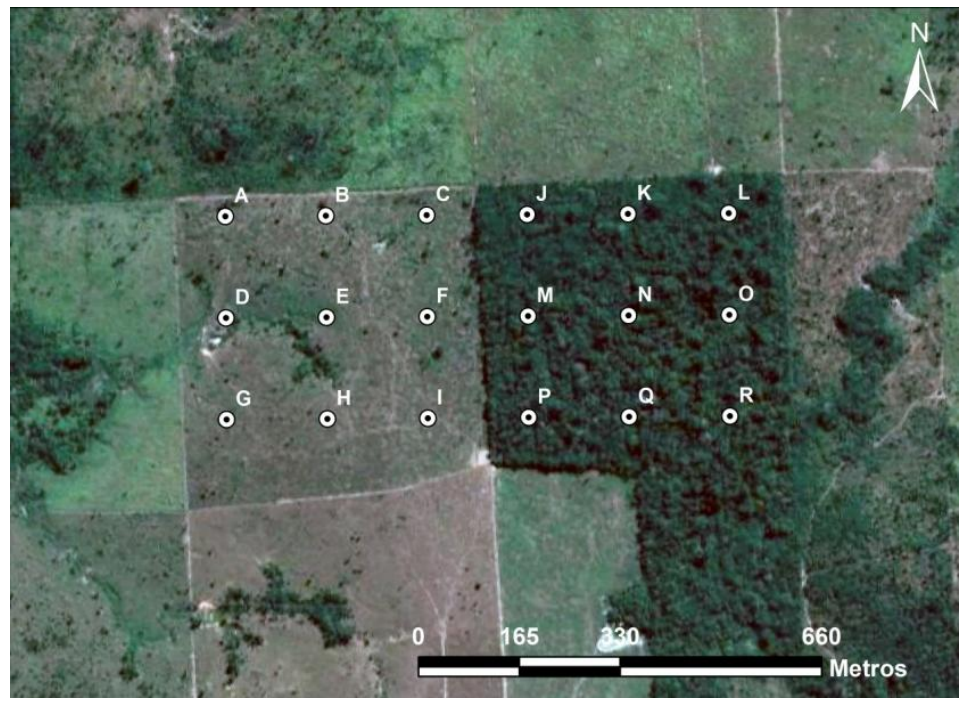

Figura 2 - Grade de pontos escolhida para amostragem, com distância aproximada de 165 metros entre os pontos.

Os dados obtidos para cada tipo de cobertura foram testados a aderência à normalidade, por meio do teste de Shapiro-Wilk (nível de significância de 5\%). Considerando a distribuição dos dados, avaliou-se a influência da conversão da área de Floresta Amazônica em pastagem sobre os parâmetros biofísicos por meio do teste de Mann-Whitney (nível de significância de 5\%), para os dados não normais, e pelo teste-t (nível de significância de 5\%) para os dados normais. 


\section{RESULTADOS E DISCUSSÃO}

Os valores dos parâmetros biofísicos extraídos para pastagem estão apresentados na tabela 1 , conforme a grade de pontos para ambos os períodos. $\mathrm{Na}$ tabela 2, estão apresentados os valores referentes à floresta nativa.

Tabela 1 - Valores obtidos para todos os pontos da grade escolhida para análise do talhão de pastagem.

\begin{tabular}{|c|c|c|c|c|c|c|c|c|c|c|}
\hline \multirow[b]{2}{*}{$\begin{array}{l}\text { Pon } \\
\text { tos }\end{array}$} & \multicolumn{2}{|c|}{ Localização } & \multicolumn{4}{|c|}{ Período Seco } & \multicolumn{4}{|c|}{ Período Chuvoso } \\
\hline & Lat. & Long. & Alb. & NDVI & $\begin{array}{c}\text { Temp } \\
\text { (oC) }\end{array}$ & $\begin{array}{c}R n \\
\left(W / m^{2}\right)\end{array}$ & Alb. & NDVI & $\begin{array}{c}\text { Temp } \\
\text { (oC) }\end{array}$ & $\begin{array}{c}R n \\
\left(W / m^{2}\right)\end{array}$ \\
\hline A & $-9,3660$ & $-59,2455$ & 0,24 & 0,70 & 28,38 & 596,70 & 0,21 & 0,85 & 26,40 & 555,87 \\
\hline B & $-9,3660$ & $-59,244$ & 0,24 & 0,73 & 28,24 & 600,88 & 0,22 & 0,88 & 26,45 & 548,75 \\
\hline C & $-9,3660$ & $-59,2425$ & 0,23 & 0,77 & 28,08 & 604,74 & 0,21 & 0,88 & 26,46 & 556,54 \\
\hline D & $-9,3675$ & $-59,2455$ & 0,23 & 0,63 & 28,34 & 603,00 & 0,19 & 0,79 & 26,51 & 571,96 \\
\hline $\mathbf{E}$ & $-9,3675$ & $-59,244$ & 0,24 & 0,74 & 28,15 & 601,92 & 0,20 & 0,85 & 26,46 & 559,75 \\
\hline $\mathbf{F}$ & $-9,3675$ & $-59,2425$ & 0,24 & 0,78 & 28,07 & 602,00 & 0,21 & 0,87 & 26,42 & 556,40 \\
\hline G & $-9,3690$ & $-59,2455$ & 0,24 & 0,79 & 28,33 & 596,99 & 0,21 & 0,82 & 26,56 & 556,09 \\
\hline $\mathbf{H}$ & $-9,3690$ & $-59,244$ & 0,24 & 0,79 & 28,12 & 599,81 & 0,20 & 0,82 & 26,51 & 558,43 \\
\hline \multirow[t]{2}{*}{ I } & $-9,3690$ & $-59,2425$ & 0,24 & 0,78 & 28,09 & 597,18 & 0,20 & 0,87 & 26,48 & 559,63 \\
\hline & \multicolumn{2}{|c|}{ Valores Médios } & 0.24 & 0,75 & 28,20 & 600,36 & 0,20 & 0,85 & 26,47 & 558,16 \\
\hline \multicolumn{3}{|c|}{ Mediana } & 0,24 & 0,77 & 28,15 & 600,88 & 0,21 & 0,85 & 26,46 & 556,54 \\
\hline \multicolumn{3}{|c|}{ Desvio Padrão } & $\pm 0,00$ & $\pm 0,05$ & $\pm 0,12$ & $\pm 2,89$ & $\pm 0,01$ & $\pm 0,03$ & $\pm 0,05$ & $\pm 6,11$ \\
\hline
\end{tabular}

Tabela 2 - Valores obtidos para todos os pontos da grade escolhida para análise do talhão de floresta nativa.

\begin{tabular}{ccccccccccc}
\hline & \multicolumn{2}{c}{ Localização } & \multicolumn{4}{c}{ Período Seco } & \multicolumn{3}{c}{ Período Chuvoso } \\
\hline $\begin{array}{c}\text { Pon } \\
\text { tos }\end{array}$ & Lat. & Long. & Alb. & NDVI & $\begin{array}{c}\text { Temp } \\
(\mathbf{( o C})\end{array}$ & $\begin{array}{c}\text { Rn } \\
\left(\mathbf{W} / \mathbf{m}^{2}\right)\end{array}$ & Alb. & NDVI & $\begin{array}{c}\text { Temp } \\
(\mathbf{0})\end{array}$ & $\begin{array}{c}\text { Rn } \\
\left(\mathbf{W} / \mathbf{m}^{2}\right)\end{array}$ \\
\hline J & $-9,3660$ & $-59,2410$ & 0,22 & 0,88 & 27,65 & 621,38 & 0,18 & 0,92 & 26,31 & 575,87 \\
K & $-9,3660$ & $-59,2395$ & 0,23 & 0,89 & 27,50 & 614,91 & 0,16 & 0,90 & 26,33 & 576,30 \\
L & $-9,3660$ & $-59,238$ & 0,21 & 0,88 & 27,65 & 627,21 & 0,17 & 0,91 & 26,29 & 584,00 \\
M & $-9,3675$ & $-59,241$ & 0,21 & 0,87 & 27,68 & 627,49 & 0,17 & 0,90 & 26,29 & 583,83 \\
N & $-9,3675$ & $-59,2395$ & 0,21 & 0,88 & 27,50 & 628,08 & 0,17 & 0,90 & 26,26 & 592,16 \\
- & $-9,3675$ & -59.238 & 0,22 & 0,88 & 27,52 & 618,31 & 0,17 & 0,90 & 26,32 & 584,45 \\
P & $-9,3690$ & $-59,241$ & 0,21 & 0,88 & 27,89 & 627,44 & 0,16 & 0,91 & 26,35 & 590,40
\end{tabular}




\begin{tabular}{|c|c|c|c|c|c|c|c|c|c|c|}
\hline $\mathbf{Q}$ & $-9,3690$ & $-59,2395$ & 0,21 & 0,87 & 27,67 & 631,10 & 0,16 & 0,90 & 26,29 & 591,98 \\
\hline \multirow[t]{4}{*}{$\mathbf{R}$} & $-9,3690$ & -59.238 & 0,21 & 0,87 & 27,57 & 627,61 & 0,16 & 0,91 & 26,34 & 597,00 \\
\hline & \multicolumn{2}{|c|}{ Valores Médios } & 0,21 & 0,88 & 27,62 & 624,84 & 0,17 & 0,91 & 26,31 & 586,22 \\
\hline & \multicolumn{2}{|c|}{ Mediana } & 0,21 & 0,88 & 27,65 & 627,44 & 0,17 & 0,90 & 26,31 & 584,45 \\
\hline & \multicolumn{2}{|c|}{ Desvio Padrão } & $\pm 0,01$ & $\pm 0,01$ & $\pm 0,12$ & $\pm 5,36$ & $\pm 0,01$ & $\pm 0,01$ & $\pm 0,03$ & $\pm 7,26$ \\
\hline
\end{tabular}

\subsection{ANÁLISE ESTATÍSTICA}

Os níveis de significância obtidos pelo teste de Shapiro-Wilk estão na Tabela 3. Conforme os níveis de significância dados pelo teste de Mann-Whitney e teste-t ( $p$-valores menores que 0,05 ), houve diferença significativa, em ambas estações, entre os índices de albedo, NDVI, temperatura e saldo de radiação da área de pastagem e de floresta. Isto é, a conversão de floresta em pastagem pode alterar significativamente os parâmetros biofísicos de uma região, podendo, assim, como consequência, alterar o microclima local.

Tabela 3 - Resultado dos testes de Shapiro-Wilk, para a testar a normalidade, e do teste-t e de Mann-Whitney para comparação entre os índices biofísicos das duas áreas (pastagem e floresta). Nível de significância de 5\%. S e C representam respectivamente os períodos seco e chuvoso.

\begin{tabular}{ccccc}
\hline Parâmetros & N & Shapiro-Wilk (Sig) & Mann-Whitney (Sig) & Teste-t (Sig) \\
\hline Albedo (S) & 9 & 0,001 & 0,000 & - \\
NDVI (S) & 9 & 0,010 & 0,000 & - \\
Temperatura (S) & 9 & 0,000 & 0,000 & - \\
Rn (S) & 9 & 0,006 & 0,000 & - \\
\hline Albedo (C) & 9 & 0,019 & 0,000 & - \\
NDVI (C) & 9 & 0,023 & 0,000 & 0,000 \\
Temperatura (C) & 9 & 0,178 & - & 0,000 \\
\hline Rn (C) & 9 & 0,050 & - &
\end{tabular}

\subsection{ALBEDO DE SUPERFÍCIE}

Os valores de albedo obtidos para cada ponto analisado (especificados na Figura 2), tanto para pastagem quanto para floresta, bem como para o período seco e chuvoso (apresentados na Tabela 1 e 2), apresentaram insignificante diferença, com desvio padrão de aproximadamente $\pm 0,01$, ou seja, os valores de albedo podem ser considerados homogêneos dentro da área correspondente.

Desta forma, de acordo com a Figura 3, a conversão da floresta nativa (à direita na área de estudos, representados pelos pontos de J até R na Figura 2), em pastagem (à esquerda na área de estudos, referentes aos pontos $A$ até I na Figura 2), resultou em um aumento médio no albedo de, aproximadamente, 
$11 \%$ (de 0,21 para 0,23) para o período seco, contra aproximadamente $21 \%$ (de 0,17 para 0,20) no período chuvoso.

Já em relação a temporariedade, o valor de albedo no talhão de floresta aumentou cerca de $21 \%$ (de 0,17 para 0,21 ) do período chuvoso em relação ao período seco. Esse aumento foi menor no talhão de pastagem, onde os valores aumentaram cerca de $14 \%$ (de 0,20 para 0,24).

Os maiores valores obtidos foram para a área de pastagem. Isso deve-se ao fato de que, em área de pastagem, principalmente no período seco, existe maior reflexão da radiação solar incidente quando comparada com as florestas (GASH et al., 1996). Nas florestas, o baixo albedo se deve à grande parte da radiação incidente ser capturada pelo interior do dossel antes de ser refletida (LEITÃO et al.,2002). Os resultados obtidos para região de pastagem foram semelhantes aos de Liberato (2011) que apresentou valor de 0,20 para albedo em uma área de pastagem na Reserva Biológica do Jaru-Rondônia.
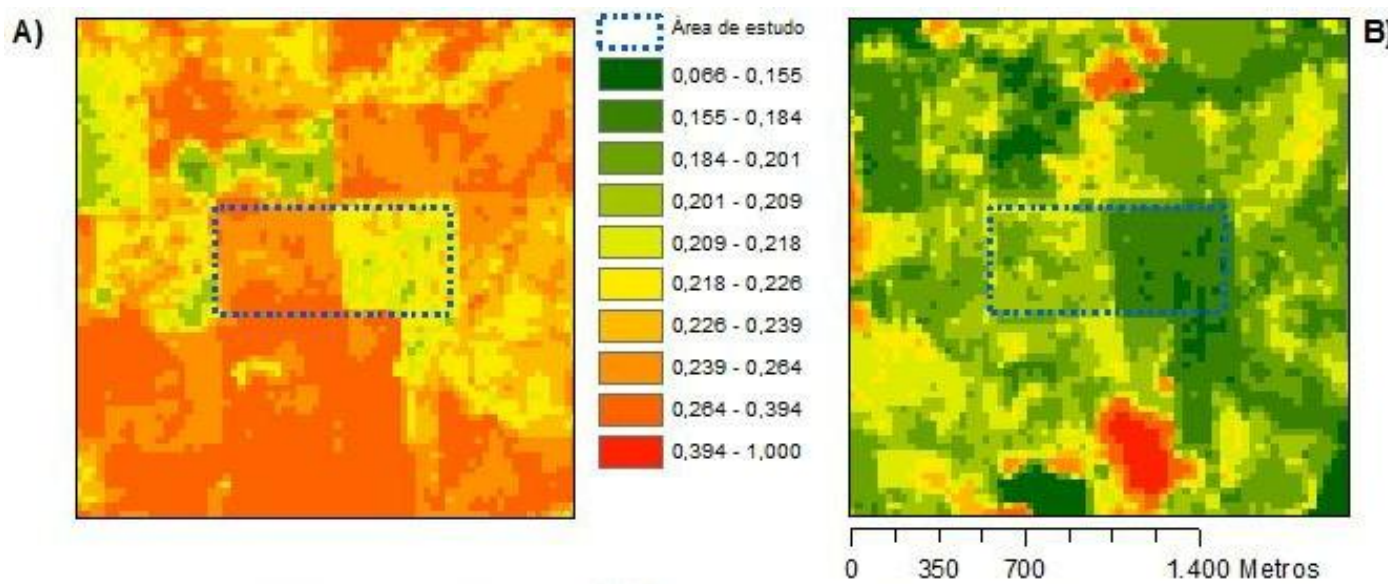

Figura 3 - Distribuição do albedo para o período seco (A) e chuvoso (B) nos talhões de pastagem, lado esquerdo, e floresta, lado direito da área de estudo localizada no município de Colniza-MT.

\section{3 ÍNDICE DE VEGETAÇÃO DA DIFERENÇA NORMALIZADA (NDVI)}

Em relação aos resultados obtidos de NDVI, foi observado que a mudança de cobertura vegetal de floresta nativa em pastagem (Figura 4) ocasionou em uma redução do NDVI, cerca de $15 \%$ (de 0,88 para 0,75) no período seco e $6 \%$ (de 0,91 para 0,85 ) no período chuvoso.

Temporalmente, os valores de NDVI no talhão de floresta nativa caíram em média $3 \%$ (de 0,88 para 0,91) entre o período chuvoso e o seco. Já para o talhão de pastagem, a queda de NDVI do período chuvoso para o seco foi mais brusca, em cerca de $14 \%$ (de 0.85 para 0.75 ). Essa pequena variação sazonal encontrada na floresta nativa se deve às características da vegetação amazônica, considerada como "sempre verde", pois esta sofre pouco no período de estiagem, o que explica essa pequena variação no NDVI entre os períodos analisados (PAVÃO, 2016). 
A)

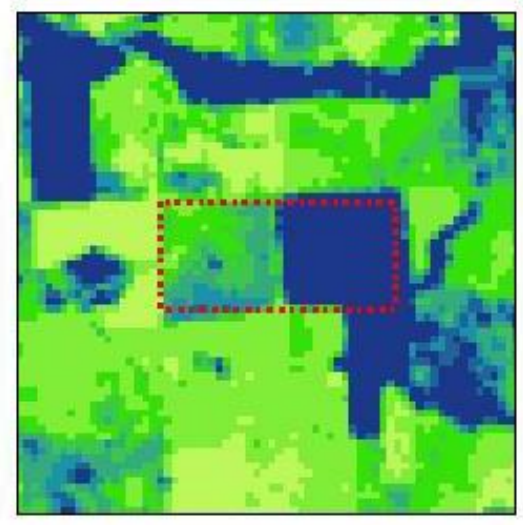

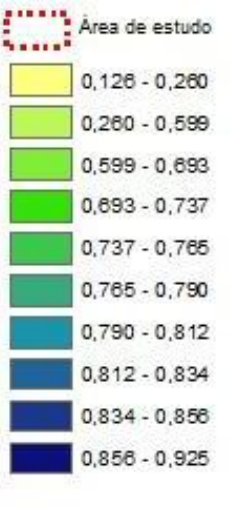

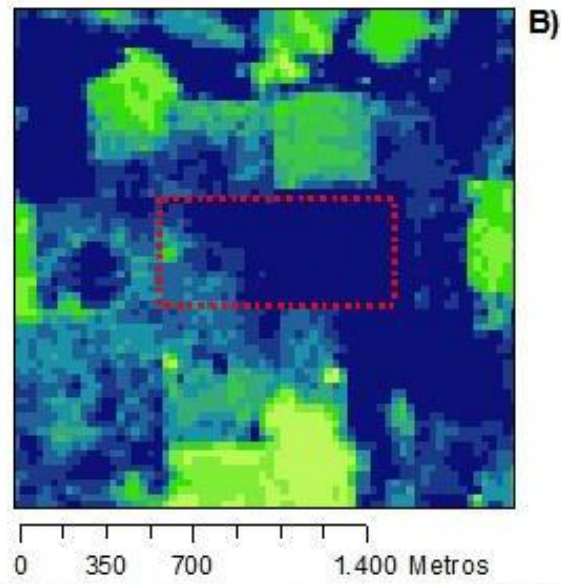

Figura 4 - Distribuição do NDVI para o período seco (A) e chuvoso (B) nos talhões de pastagem, lado esquerdo, e floresta, lado direito, da área de estudo localizada no município de Colniza-MT.

\subsection{TEMPERATURA DE BRILHO}

Na Figura 5 é possível observar que houve um pequeno aumento da temperatura de brilho da pastagem em relação a floresta nativa, sendo este aumento de, aproximadamente, $2 \%$ (de $27,62^{\circ} \mathrm{C}$ para $28,20^{\circ} \mathrm{C}$ ) para o período seco e $1 \%$ (de $26,31^{\circ} \mathrm{C}$ para $26,47^{\circ} \mathrm{C}$ ) para o período chuvoso. Esse aumento na temperatura pode promover uma redução na umidade relativa do ar, especialmente nas estações mais secas do ano e intensificar o risco às queimadas (SAUSEN \& LACRUZ, 2015).

A)

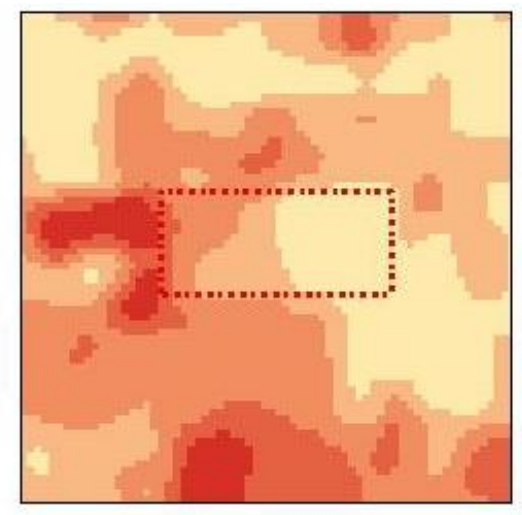

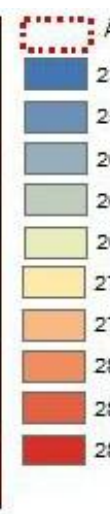

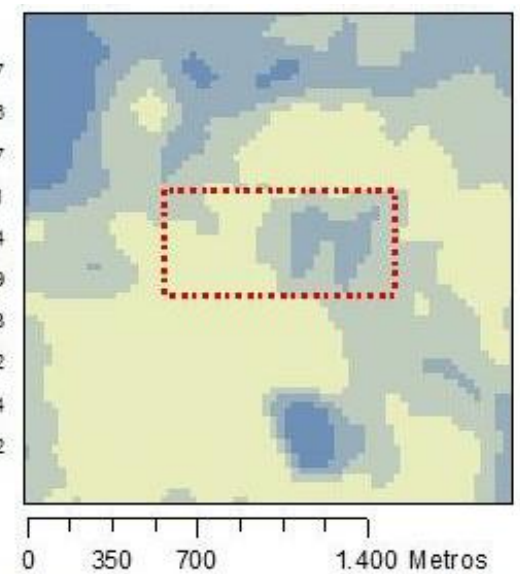

B)

Figura 5 - Distribuição da Temperatura de Brilho para o período seco (A) e chuvoso (B) nos talhões de pastagem, lado esquerdo, e floresta, lado direito, da área de estudo localizada no município de Colniza-MT.

Quanto a temporariedade, do período chuvoso para o seco, houve aumento de $6 \%$ (de $26,47^{\circ} \mathrm{C}$ para $28,20^{\circ} \mathrm{C}$ ) da temperatura na pastagem e de $5 \%$ (de $26,31^{\circ} \mathrm{C}$ para $27,62^{\circ} \mathrm{C}$ ) na floresta nativa. Essa menor variação da temperatura na floresta nativa se deve a cobertura vegetal constante, mantendo uma temperatura mais baixa em relação a pastagem. Já que em áreas de 
pastagens, por evidenciarem baixa densidade de vegetação, a energia disponível ao ambiente é utilizada principalmente para aquecimento do solo e do ar adjacente à superfície, portanto, resulta em maiores valores de temperatura de brilho da superfície (BIUDES et al., 2009; PAVÃO et al., 2017). Observa-se, também, que os dados obtidos para temperatura de brilho e NDVI apresentaram correlação negativa, com nível de significância de 1\% (Figura 6 e Figura 7).

Os coeficientes de correlação de Spearman (rô de Spearman) obtidos entre temperatura de brilho e NDVI foram de $-0,89$ e $-0,80$ para os períodos seco e chuvoso, respectivamente. Com isso, pode-se dizer que ambos estão inversamente correlacionados e, por isso, para maiores valores de NDVI têm-se menores valores de temperatura. Na floresta, por exemplo, por ter uma vegetação mais densa e NDVI alto, a energia disponível é partilhada no processo de evapotranspiração e que, consequentemente, acaba resfriando a superfície vegetada (BIUDES et al., 2009; PAVÃ̃ et al.,2015).

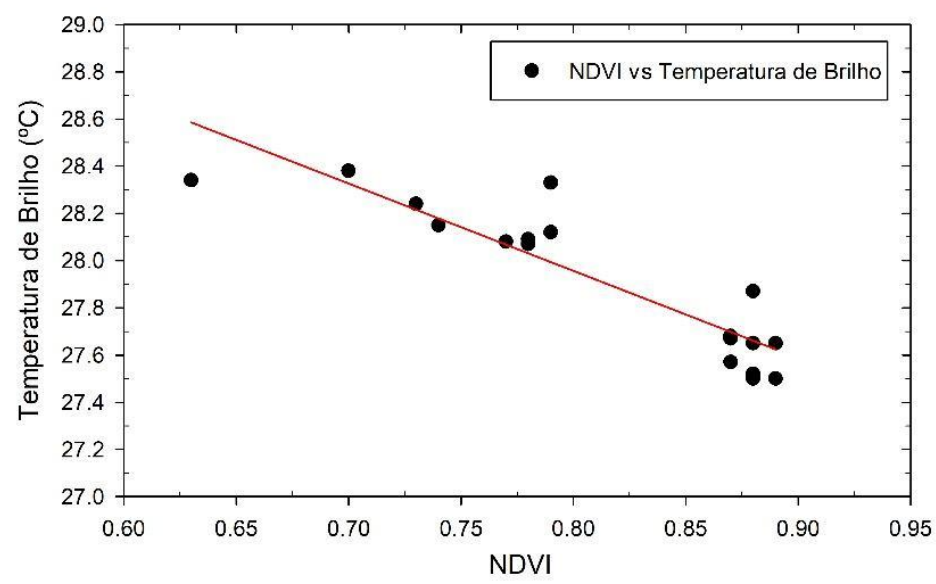

Figura 6 - Correlação significativa dos dados de temperatura de brilho e NDVI para o período seco. Rô de Spearman=-0,89 ( $p$-valor menor que 0,01).

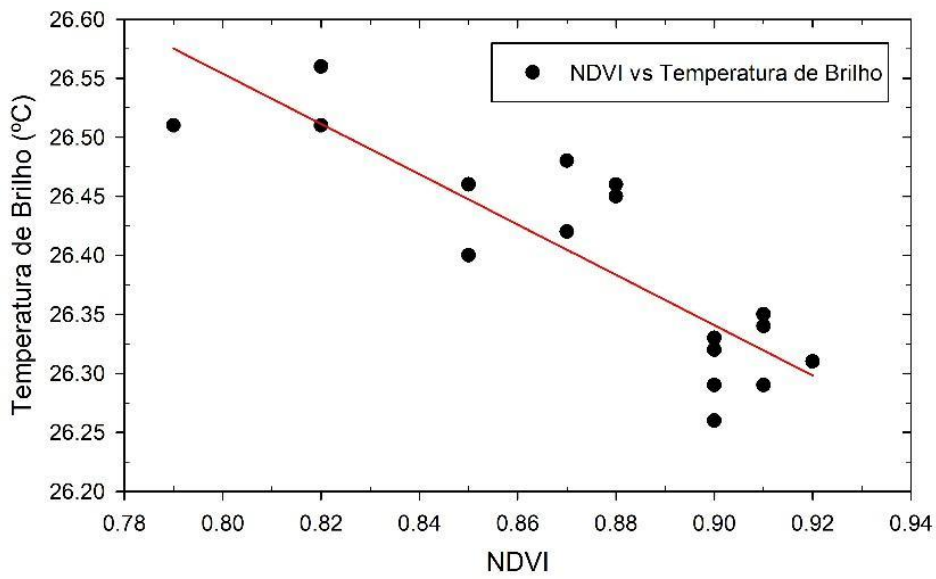

Figura 7 - Correlação significativa dos dados de temperatura de brilho e NDVI para o período chuvoso. Rô de Spearman=-0,80 ( $p$-valor menor 0,01). 


\subsection{SALDO DE RADIAÇÃO}

Na Figura 8, tem-se uma amostragem espacial dos valores de Saldo de Radiação (Rn), onde podemos perceber maiores resultados para $\mathrm{Rn}$, em ambos os períodos, para o talhão de floresta nativa. O valor médio de saldo de radiação obtido para o talhão de pastagem, no período seco (lado A da Figura 7), foi de $600,36 \mathrm{Wm}-2$ contra 624,84 Wm-2 na floresta nativa (lado B da Figura 7), 0 que caracteriza um aumento de $4 \%$. Já em relação ao período chuvoso, foram obtidos, em média, $558,16 \mathrm{Wm}-2$ para o talhão de pastagem e 586,22 Wm-2 para floresta nativa, sendo um aumento de cerca de $5 \%$.

Como o saldo de radiação é o resultado da inserção de todas as variáveis analisadas (SILVA et al., 2015), na área que se obteve menor albedo e maior NDVI teve-se um maior valor de Rn (floresta nativa), tanto no período seco quanto no período chuvoso. Assim, o resultado obtido neste trabalho corrobora com os resultados de Silva et al. (2015) e Pavão et al. (2016) que obtiveram maiores valores de saldo de radiação em regiões de Floresta Amazônica quando comparados com regiões de pastagens.

Quando analisado temporalmente, em relação aos períodos seco e chuvoso, os resultados obtidos permitem observar pouca variação de saldo de radiação. Tratando-se, em ambas as áreas, do período chuvoso ao período seco, houve um aumento de $7 \%$ nos valores de Rn na pastagem e de $6 \%$ na floresta nativa. Estes resultados corroboram com os encontrados por Feitosa (1998) e Andrade (2009), que também encontraram maiores valores de $\mathrm{Rn}$ em períodos considerados secos.

A)

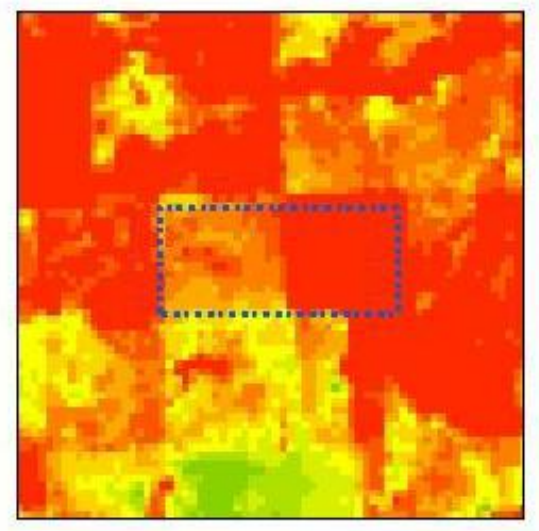

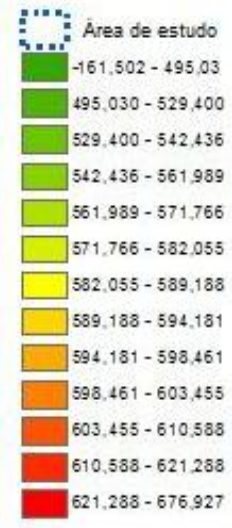

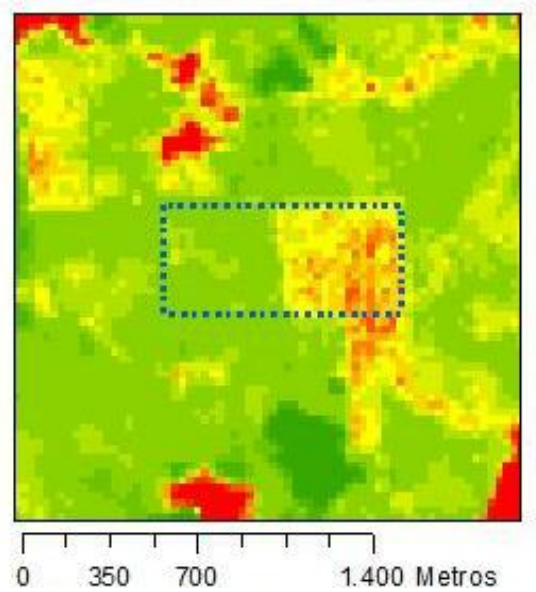

Figura 8 - Distribuição do Saldo de Radiação para o período seco (A) e chuvoso (B) nos talhões de pastagem, lado esquerdo, e floresta, lado direito, da área de estudo localizada no município de Colniza-MT.

\section{CONCLUSÃO}

Os resultados indicam que a alteração da cobertura de floresta nativa em pastagem afeta diretamente os parâmetros biofísicos. Essa mudança ocasionou um aumento médio no albedo de $11 \%$ para o período seco e de $21 \%$ para o período chuvoso, sendo as maiores médias observadas na estação seca para as duas áreas. 
Os menores valores de NDVI foram observados para a pastagem em ambos os períodos, e sazonalmente o NDVI apresenta maiores valores para o período chuvoso, o que se deve ao desenvolvimento vegetativo em decorrência da disponibilidade hídrica.

O NDVI e a temperatura de brilho mostraram-se ser inversamente correlacionados, tendo assim, as maiores temperaturas em locais onde há menores densidade de vegetação, resultando, por fim, em um saldo de radiação menor no talhão de pastagem em comparação a área de floresta nativa.

O talhão de floresta nativa registrou os maiores valores para o saldo de radiação em ambos os períodos, e temporalmente os maiores valores foram registrados durante o período seco. Estes resultados indicam que a substituição da vegetação nativa por pastagem resulta em menor absorção da radiação solar pela superfície, acarretando em um menor saldo de radiação na região desmatada.

\section{AGRADECIMENTOS}

Os autores agradecem à Coordenação de Aperfeiçoamento de Pessoal de Nível Superior (CAPES) e ao Conselho Nacional para o Desenvolvimento Científico e Tecnológico ( $\mathrm{CNPq}$ ), pelo apoio financeiro e ao Programa de PósGraduação em Física Ambiental (PPGFA) da Universidade Federal de Mato Grosso pelo incentivo a pesquisa.

\section{REFERÊNCIAS}

ALLEN, R. G.; TASUMI, M.; TREZZA, R. Satellite-based energy balance for mapping evapotranspiration with internalized calibration (METRIC)-Model. Journal of irrigation and drainage engineering, v. 133, n. 4, p. 380-394, 2007.

ALLEN, R.; BASTIAANSSEN, W.; WATERS, R.; TASUMI, M.; TREZZA, R. Surface energy balance algorithms for land (SEBAL). Idaho implementation-Advanced training and user's manual, v. 1, 2002.

ALVARES, C. A.; STAPE, J. L.; SENTELHAS, P. C.; MORAES, G.; LEONARDO, J.; SPAROVEK, G. Koppen's climate classification map for Brazil. Meteorologische Zeitschrift, v. 22, n. 6, p. 711-728, 2013.

ANDRADE, N. L.; AGUIAR, R. G.; SANCHES, L.; ALVES, E. C. R. F.; NOGUEIRA, J. S.; Partição do Saldo de radiação em áreas de floresta amazônica e floresta de transição amazônia-cerrado. Revista Brasileira de Meteorologia, v.24, n.3, 346-355, 2009

ARPA. (Programa Áreas Protegidas da Amazônia). Arpa Biodiversidade. 2012. Disponível em: < http://www.programaarpa.gov.br/wpcontent/uploads/2012/10/ arpaBiodiversidade.pdf $>$. Acesso em 12 de abr. de 2017.

BASTIAANSSEN, W. G. M. SEBAL-based sensible and latent heat fluxes in the irrigated Gediz Basin, Turkey. Journal of hydrology, v. 229, n. 1, p. 87-100, 2000.

BERBET. M. L. C. Variação sazonal do albedo e sua influência na mudança do padrão de chuva, em consequência da conversão da floresta tropical em 
pastagem. Tese de Doutorado. Universidade Federal de Viçosa Programa de Pós-Graduação em Meteorologia Agrícola. VIÇOSA, MG. 47p. 2002.

BERNARDES, T.; ADAMI, M.; FORMAGGIO, A. R.; MOREIRA, M. A.; FRANÇA, D. A.; NOVAES, M. R. Imagens mono e multitemporais Modis para estimativa da área com soja no estado de Mato Grosso. Pesquisa Agropecuária Brasileira, v. 46 , n. 11, p. 1530-1537, 2011.

BEZERRA, J. M.; MOURA, G. B. A.; SILVA, B. B.; LOPES, P. M. O.; SILVA, E. F. F. Parâmetros biofísicos obtidos por sensoriamento remoto em região semiárida do estado do Rio Grande do Norte, Brasil. Revista Brasileira de Engenharia Agrícola e Ambiental, v. 18, n. 1, p. 73-84, 2014.

BIUDES, M. S.; CAMPELO JUNIOR, J. H.; NOGUEIRA, J. S.; SANCHES, L. Estimativa do balanço de energia em cambarazal e pastagem no norte do Pantanal pelo método de razão de Bowen. Revista Brasileira de Meteorologia, v.24, n.2, p. 135 - 143, 2009.

CUNHA, J. E. B. L.; RUFINO, I. A. A.; SILVA, B. B.; CHAVES, I. B. Dinâmica da cobertura vegetal para a Bacia de São João do Rio do Peixe, PB, utilizando-se sensoriamento remoto. Revista Brasileira de Engenharia Agrícola e Ambiental, v.16, p.539-548, 2012.

DUARTE, H. F.; DIAS, N. L.; MAGGIOTTO, S. R. Assessing daytime downward longwave radiation estimates for clear and cloudy skies in Southern Brazil. Agricultural and forest meteorology, v. 139, n. 3, p. 171-181, 2006.

FAUSTO, M. A.; ANGELINI, L. P.; MARQUES, H. O.; FILHO, A. S.; MACHADO, N. G.; BIUDES, M. S. Impacto da alteração do uso do solo no saldo de radiação no Cerrado do sul de Mato Grosso. Ambiente \& Água. p. 350-361, 2016.

FEITOSA, J. R. P.; COSTA, R. F.; FISCH, G.; SOUZA, S. D.; NOBRE, C. A. Radiação solar global em áreas de floresta e pastagem na Amazônia. Revista Brasileira de Agrometeorologia, v. 6, n. 1, p. 1-7, 1998.

GASH, J.H.C.; NOBRE, C.A.; ROBERT, J.M.; VICTORIA, R.L. Amazonian deforestation and climate. Wiley, Chichester, 595p. 1996.

GUERRA, Wilson Barbosa. O Brasil e a segurança do Atlântico Sul. Nação e Defesa, 2011.

GUILHERME, A. P.; MOTA, A. B. S.; MOTA, D. S.; MACHADO, N. G.; BIUDES, M. S. Uso de índice de vegetação para caracterizar a mudança no uso do solo em Coari-AM/Use of vegetation index to characterize the land use change in CoariAM. Revista Sociedade \& Natureza, v. 28, n. 2, 2016.

KENNEDY, R.; SCAMBOS, T. A.; SCHAAF, C. B.; SCHOTT, J. R.; SHENG, Y.; VERMOTE, E. F.; BELWARD, A. S.; BINDSCHADLER, R.; COHEN, W. B.; GAO, F.; HIPPLE, J. D.; HOSTERT, P.; HUNTINGTON, J.; JUSTICE, C. O.; KILIC, A.; KOVALSKYY, V.; LEE, Z. P.; LYMBURNER, L.; MASEK, J. G.; MCCORKEL, J.; SHUAI, Y.; TREZZA, R.; VOGELMANN, J.; WYNNE, R. H.; ZHU, Z. Landsat-8: Science and product vision for terrestrial global change research. Remote Sensing of Environment, v. 145, p. 154-172, 2014.

LEE, D. S.; STOREY, J. C.; CHOATE, M. J.; HAYES, R. W. Four years of Landsat7 on-orbit geometric calibration and performance. IEEE Transactions on Geoscience and Remote Sensing, v. 42, n. 12, p. 2786-2795, 2004. 
LEITÃO, M. D. M., SANTOS, J. M., \& OLIVEIRA, G. M. Estimativas do albedo em três ecossistemas da floresta amazônica. Revista Brasileira de Engenharia Agrícola e Ambiental, v.6, n.2, p.256-261. 2002.

LIBERATO, A. M. Albedo à superfície a partir de imagens LANDSAT 5-TM em áreas de floresta e pastagem na Amazônia. Revista de Geografia (Recife). ISSN: 2238-6211, v. 28, n. 1, p. 110-119, 2011.

LIMA, M. O. Amazônia, uma história de impactos e exposição ambiental em paralelo à instalação de grandes empreendimentos na região. Revista PanAmazônica de Saúde, v. 7, n. 2, p. 9-11, 2016.

LOPES, H. L.; CANDEIAS, A. L. B.; ACCIOLY, L. J. O.; SOBRAL, M. DO C. M.; PACHECO, A. P. Parâmetros biofísicos na detecção de mudanças na cobertura e uso do solo em bacias hidrográficas. Revista Brasileira de Engenharia Agrícola e Ambiental, v.14, p.1210-1219, 2010.

MARTINS, A. L.; CUNHA, C. R.; PEREIRA, V. M. R.; DANELICHEN, V. H. M.; MACHADO, N. G.; LOBO, F. A.; MUSIS, C. R.; BIUDES, M. S. Mudanças em índices biofísicos devido à alteração da cobertura do solo em área nativa de Cerrado em Mato Grosso. Ciência e Natura, v. 37, n. 4, p. 152-159, 2015.

OLIVEIRA, L. M. M.; MONTENEGRO, S. M. G. L.; ANTONINO, A. C. D.; SILVA, B. B.; MACHADO, C. C. C.; GALVÍNCIO, J. D. Análise quantitativa de parâmetros biofísicos de bacia hidrográfica obtidos por sensoriamento remoto. Pesquisa Agropecuária Brasileira, v. 47, n. 9, p. 1209-1217, 2012.

PAVÃO, V. M.; NASSARDEN, D. C. S.; PAVÃO, L. L.; MACHADO, N. G.; BIUDES, M. S. Impacto da Conversão da Cobertura Natural em Pastagem e Área Urbana sobre Variáveis Biofísicas no Sul do Amazonas. Revista Brasileira de Meteorologia, v. 32, n. 3, p. 343-351, 2017.

PAVÃO, V. M.; QUERINO, C. A. S.; BENEDITTI, C. A.; PAVÃO, L. L.; QUERINO, J. K. A. S.; MACHADO, N. G.; BIUDES, M. S. Variação Espacial e Temporal do Saldo de Radiação Superficial em uma área do Sul do Amazonas, Brasil. Ra'e Ga, v. 37, p.333-352, Curitiba. 2016.

PAVÃO, V. M.; QUERINO, C.; BENEDITTI, C.; PAVÃO, L.; QUERINO, J.; MACHADO, N. Temperatura e albedo da superfície por imagens TM Landsat 5 em diferentes usos do solo no sudoeste da Amazônia brasileira. Revista Brasileira de Climatologia, v. 16, p. 169-183, 2015.

PEREIRA, G.; MORAES, E. C.; ARAI, E.; OLIVEIRA, L. D. Estudo preliminar da estimativa da reflectância e albedo de microssistemas pantaneiros a partir de imagens de satélite. Revista Brasileira de Cartografia, v. 59, n. 1, p. 55-61, 2007.

PISANI, R. J.; ZIMBACK, C. R. L.; CAMPOS, S.; BARROS, Z. X. Relações entre o método MAXVER e NDVI para classes de uso do solo na sub-bacia do Rio das Pedras Itatinga - SP. Revista Científica Eletrônica de Agronomia, Garça, v. 22, n. 2, p. 1-12, 2012.

PONZONI F.J.; SHIMABUKURO, Y.E. Sensoriamento Remoto no estudo da vegetação. São José dos Campos: Parêntese. 136 p. 2009. 
RIBEIRO, E. P.; NÓBREGA S. R.; MOTA FILHO F. O.; MOREIRA E. B. M. Parâmetros biofísicos na detecção de mudanças ambientais na bacia hidrográfica do rio Pajeú. Revista de Geografia (UFPE), v. 32, n. 1, p. 221-246, 2015.

ROCHA, VINÍCIUS MACHADO; SILVA CORREIA, F. W.; MORELLI FONSECA, P. A. Reciclagem de precipitação na Amazônia: um estudo de revisão. Revista Brasileira de Meteorologia, v. 30, n. 1, p. 59-70, 2015.

Rouse Jr, J., Haas, R. H., Schell, J. A., \& Deering, D. W. Monitoring vegetation systems in the Great Plains with ERTS.1974.

SILVA, B. B. D. ; BRAGA, A. C.; BRAGA, C. C.; OlIVEIRA, L. M.; MONTENEGRO, S. M.; BARBOSA JUNIOR, B. Procedures for calculation of the albedo with OLILandsat 8 images: Application to the Brazilian semi-arid. Revista Brasileira de Engenharia Agrícola e Ambiental, v. 20, n. 1, p. 3-8, 2016.

SILVA, B. B.; BRAGA, A. C.; BRAGA, C. C. Balanço de radiação no perímetro irrigado São Gonçalo - PB mediante imagens orbitais. Revista Caatinga, v. 24, p. 145-152, 2011.

SILVA, L. C.; CUNHA, J. M.; MACHADO, N. G.; CAMPOS, M. C. C.; BIUDES, M. S. Estimativa do Balanço de Radiação por Sensoriamento Remoto de diferentes usos de solo no Sudoeste da Amazônia Brasileira. Soc. \& Nat., Uberlândia, 27 (2): 341-356, 2015.

STOREY, J.; CHOATE, M.; LEE, K. Geometric performance comparison between the OLI and the ETM+. Proceedings of the PECORA. 2008.

TEIXEIRA, A. H. DE C.; BASTIAANSSEN, W. G. M.; AHMAD, M. D.; BOS, M. G. Reviewing SEBAL input parameters for assessing evapotranspiration and water productivity for the LowMiddle São Francisco River basin, Brazil Part B: Application to the large scale. Agricultual and Forest Meteorology, v.149, p.477490, 2009.

TRES, A.; TETTO, A. F.; SOARES, V. R.; SOARES, R. V.; WEN DLING, W. T.; MENGATTO, A. P. R. Classificação Do Estado De Mato Grosso Segundo Sistema De Zonas De Vida De Holdridge. Enciclopédia Biosfera, v.13, n.23, p. 329-343, 2016.

WMO. Manual on the Global Observing System. World Meteorological Organization. Geneva, p. 50. 2003. (ISBN 92-63-13544-4). 